\title{
FAST NMPC OF A DC-DC CONVERTER: AN EXACT NEWTON REAL-TIME ITERATION APPROACH
}

\author{
J. Bonilla* R. De Keyser** M. Diehl* \\ J. Espinosa ${ }^{* * *}$ \\ * Research Group SCD-SISTA and OPTEC, Electrical \\ Engineering Department, Katholieke Universiteit Leuven, \\ Kasteelpark Arenberg 10, B-3001 Leuven, Belgium \\ ** Electrical energy, Systems and Automation Department \\ (EeSA), Universiteit Gent, Technologiepark 913, 9052 \\ Ghent-Zwijnaarde, Belgium \\ *** Faculty of Mines (GAUNAL), National University of \\ Colombia, Av. 80 N.65-223 Medellín,Colombia
}

\begin{abstract}
This paper presents a Newton optimization technique applied to the Nonlinear Model Predictive Controller (NMPC) for a DC-DC converter. The nonlinear predictor, used in this NMPC algorithm, is approximated by means of a dynamic model which is cubic in the input. This approximation allows the formulation of an explicit MPC law in terms of the states of the model. The proposed controller is tailored for this specific application, taking into account simplicity in the calculation of the control law. The approach followed makes the control law easy to implement and able to deal with the fast response dynamics presented by electronic drivers. The performance of the proposed controller is evaluated under simulation and compared with the response presented by a linear MPC. Although robustness and tracking test are performed over the whole operating range, stability results for the nonlinear MPC are not addresed.
\end{abstract}

Copyright $\odot 2007$ IFAC

Keywords: Predictive control, Nonlinear systems, Electronic applications

\section{INTRODUCTION}

Power electronic devices are nowadays applied to different areas, from power generation and distribution to embedded solutions for communication, automotive and computers industries. This wide range of applications has motivated this field to be an active research area where several topologies and control strategies have been investigated. Among all these topologies, the DC-DC boost converter presents interesting challenges from the control point of view. It exhibits a non-linear, hybrid behavior (including continuous and logical variables), non-minimum phase and additionally, very short time responses requiring nonlinear and fast control strategies which have to be implemented in real-time. However, in spite of this challenging features, boost converters are usually regulated by simple linear analog techniques based on a linear low frequency approximation of the system. Nevertheless the performance of the closed loop system can be improved by the use of nonlinear control, especially if the circuit has to apply a wide-range time-varying output voltage. A number of non-linear control techniques has been reported in the literature, such as: sliding mode controllers (Silva, 1999), (Escobar et al., 1997), 
non-linear PI methods based on extended linearization (Sira-Ramirez, 1990) and model based predictive controllers (Ramirez-Garcia and De Keyser, 2001), (Geyer et al., 2005) among others.

The paper is organized as follows: in section 2 , the hybrid model is formulated for a real low power boost converter. Section 3 shows the development of a linear MPC algorithm for a linear simplified State Space Average (SSA) model, while section 4 deals with the NMPC design for a nonlinear SSA model. The designed controllers are evaluated in section 5 and the paper is concluded in section 6 .

\section{SYSTEM MODEL}

The modeling of switched power drives can be classified depending on whether the switching signal $q(t)$ is directly manipulated ${ }^{1}$ or a pulse width modulator (PWM) is needed to drive the state of the switch.

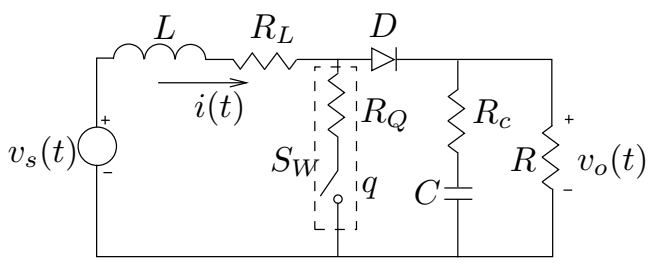

Fig. 1. Boost converter circuit

Figure 1 shows the electric circuit for a boost converter assuming internal losses in the components. It is assumed in general that $v_{o}(t)>v_{s}(t)$. Losses in the diode will be represented by its internal resistance $R_{D}$ and its forward voltage in conduction state $V_{D}$. The system is considered as an hybrid one, where $q$ is a logic variable commanding the switch state while $v_{s}(t), i(t)$ and $v_{o}(t)$ are continuous signals. A brief explanation of the boost behavior is given below

When the switch in figure 1 is closed, the diode turns off and the current circulating through the input loop loads the coil. At the same time, the capacitor feeds the load resistor with its stored charge. On the other hand, if the switch is opened, the diode turns on allowing that the energy stored in the coil circulates towards the output loop. The generated input current charges the capacitor and feeds the load resistor. If the switch is opened and closed periodically this behavior is repeated each switching cycle. This normal operation of the converter is called Continuous Conduction Mode $(C C M)$ because the current flows continuously trough the coil in both, on and off states.

A second behavior can appear when the switch is opened and all the energy in the coil is delivered

1 This approach leads to a hybrid model formulation to the output loop. At that moment the input current goes to zero and although the switch is open, the diode is not active due to the absence of current through the coil. Under this condition, the load is feeded only by the charge stored in the capacitor and the current at the input will rise only when the switch is closed again. This behavior is known as Discontinuous Conduction Mode (DCM).

Equation (1) models the behavior for the states $x(t)=\left[i(t) v_{o}(t)\right]^{T}$ in CCM as functions of the logical variable $q$, its complement $\bar{q}$ and the input voltage $v_{s}(t)$. It must be noticed that the DCM is not described by (1) but it is included in the simulator used to test the controllers.

$$
\begin{aligned}
\mathbf{x}(t) & =\mathbf{F}(q) \mathbf{x}(t)+\mathbf{G}_{\mathbf{1}}(q) v_{s}(t)+\mathbf{G}_{\mathbf{2}}(q) \\
\mathbf{F}(q)= & \left(\begin{array}{cc}
\frac{-\left(R_{L}+q R_{Q}+\bar{q} R_{D}\right)}{L} & \frac{-\bar{q}}{L} \\
\frac{\bar{q} R\left(L-C R_{C}\left(R_{L}+R_{D}\right)\right)}{L C\left(R+R_{C}\right)} & \frac{-\left(L+\bar{q} C R R_{C}\right)}{L C\left(R+R_{C}\right)}
\end{array}\right) \\
\mathbf{G}_{\mathbf{1}}(q)= & \left(\begin{array}{c}
\frac{1}{L} \\
\frac{\bar{q} C R R_{C}}{L C\left(R+R_{C}\right)}
\end{array}\right) \\
\mathbf{G}_{\mathbf{2}}(q)= & \left(\begin{array}{c}
-\bar{q} V_{D} \\
-\frac{\bar{q} V_{D} C R R_{C}}{L C\left(R+R_{C}\right)}
\end{array}\right)
\end{aligned}
$$

The system parameters, tuned for a particular setup, are summarized in table 1 .

Table 1. System Nominal Parameters

\begin{tabular}{ll}
\hline Parameter & Value \\
\hline$R$ & $162.5 \Omega$ \\
$L$ & $1.4 \mathrm{mH}$ \\
$C$ & $220 \mu \mathrm{F}$ \\
$V_{s}$ & $18.3 \mathrm{~V}$ \\
$R_{L}$ & $0.5179 \Omega$ \\
$R_{C}$ & $0 \Omega$ \\
$R_{D}$ & $0.4497 \Omega$ \\
$R_{Q}$ & $0.097 \Omega$ \\
$V_{D}$ & $0.6739 \mathrm{~V}$ \\
\hline
\end{tabular}

\section{LINEAR MPC FORMULATION}

The MPC is a group of methods with several common features. All these methods are based on the prediction of the future process outputs over a certain time horizon $N_{H}$ using a model of the process. The algorithm calculates the necessary control actions, over a control horizon $N_{u}$, in order to reach a desired trajectory using an optimization routine. This routine takes into account the predictions of the system outputs, the postulated control efforts and some constraints imposed by the designer. Only the first value of the optimal 
control sequence is applied to the process and the prediction and optimization procedures start again over the horizon shifted one sampling time ahead. This principle is called receding horizon.

Several assumptions have been made in order to design a MPC for (1). First of all, the presented hybrid system is approximated by a low frequency model (SSA model) using an PWM module to command the state of the switch and taking the average values of the states over one switching cycle $T_{s}$. The equations of the obtained model are similar to (1) but $q$ is replaced by $u(t)$ which represents the average value of the logical signal over $T_{s}$. Second, the model used for the controller design does not assume losses $\left(R_{L}=0, R_{D}=0\right.$, $R_{Q}=0, R_{C}=0$ and $\left.V_{D}=0\right)$. Finally a simple noise model to estimate the disturbances has been added. The obtained system is presented in (2).

$$
\begin{gathered}
\dot{\mathbf{x}}(t)=\mathbf{A}(\mathbf{u}) \mathbf{x}(t)+\mathbf{B}_{\mathbf{d}} d(t) \\
y(t)=\mathbf{H} \mathbf{x}(\mathbf{t}) \\
\mathbf{A}(\mathbf{u})=\left(\begin{array}{cc}
0 & -\frac{(1-u(t))}{L} \\
\frac{(1-u(t))}{C} & -\frac{1}{R C}
\end{array}\right) \\
\mathbf{B}_{\mathbf{d}}=\left(\begin{array}{c}
\frac{1}{L} \\
-K_{n}
\end{array}\right) \\
\mathbf{H}=\left(\begin{array}{ll}
0 & 1
\end{array}\right)
\end{gathered}
$$

Since the goal is to design a linear discrete controller, (2) is linearized around $v_{o}(t)=24 \mathrm{~V}$, $v_{s}(t)=18.3 \mathrm{~V}$ and used for the prediction and state estimation.

The term $d(t)$ on the right side of (2) accounts for the non-measured disturbances (such as the input voltage) and modelling mismatch. It is important to notice that the model used for the noise here is a simple integrator with output gain $B_{d}$. For this application, this gain has been designed with the following criteria: the first entry correspond to the exact effect of the disturbance $v_{s}(t)$ over the states, obtained from the simplification of (1). The sign of the second entry in $B_{d}$ comes from the negative effect of a load change on the second state as noticed in (2), its value is tuned by trial and error procedure. It must be stated that this is an initial guess for the noise model on the system, possible improvements can be achieved with a better characterization of the modelling errors.

The state observer is designed by augmenting the linearized system in order to estimate the unmeasured disturbance $d(t)$ and the input current. A Kalman filter (Kalman and Bucy, 1961) is tuned with the covariances $\left(Q_{w}\right.$ and $\left.R_{v}\right)$, providing fast disturbance rejection for the executed test.
In order to improve the response of the closed loop system with respect to output disturbances and setpoint changes, a simple first order reference filter was implemented in the setpoint $s p(t)$. The response of the controlled variable should follow the dynamics of this reference filter $r(t)$ with a time constant $\tau_{\text {ref }}$. This time constant was tuned under trial and error procedure in order to get a fast response to set point changes and preserving an stable behavior.

The designed unconstrained MPC minimizes the cost function in terms of the prediction error $\mathbf{E}(u, \mathbf{x})$ over a prediction horizon as formulated in $(3)$.

$$
\min _{u} \frac{1}{2}\|\mathbf{E}(u, \mathbf{x})\|_{2}^{2}
$$

Table 2 summarize the design parameters for the controller.

Table 2. Parameters for MPC controller

\begin{tabular}{ll}
\hline Parameter & value \\
\hline$N_{H}$ & 24 samples \\
$N_{u}$ & 1 sample \\
$\tau_{\text {ref }}$ & $2.5 \mathrm{~ms}$ \\
$T_{s}$ & $50 \mu \mathrm{s}$ \\
$K_{n}$ & 60 \\
$Q_{w}$ & 500 \\
$R_{v}$ & 0.5 \\
\hline
\end{tabular}

\section{THE NMPC}

The nonlinear version of the used MPC in this work is based on the formulation in (2). Although it is a simplified model, it preserves some of the most relevant nonlinear characteristics of the original hybrid system. For the linear model, an analytical solution to (3) is simple, since the optimization variable $u$ appears quadratically in the cost. Conversely, in the nonlinear model, an analytical solution becomes cumbersome, even for the unconstrained case. The key idea here to deal with this problem is to approximate the simplified nonlinear model by means of another nonlinear representation which can be differentiated easily.

Since the approach presented here assumes $N_{u}=$ 1 and constant disturbance over $N_{H}$, equation (2) can be easily solved over a prediction horizon in terms of the initial state $x(0), d(t)$ and $u(t)$. The estimated disturbances and the control signal are supposed to be constant over all the horizon. The analytical solution to (2), under the previous assumptions, is presented in (4).

$$
y(t)=\mathbf{G}(u, t) \mathbf{z}
$$

$$
\begin{aligned}
\mathbf{G}(u, t) & =\left(\mathbf{H} e^{\mathbf{A}(u) t}-\mathbf{H A}(u)^{-1}\left(1-e^{\mathbf{A}(u) t}\right) \mathbf{B}_{\mathbf{d}}\right) \\
\mathbf{z} & =(\mathbf{x}(0) d(t))^{T}
\end{aligned}
$$




\subsection{The polynomial approximation}

Notice that $\mathbf{G}(u, t)$ is very nonlinear in $u$. The procedure is based on an approximation of this function by a polynomial expression, which allows an easy differentiation of the prediction in terms of optimization variable. Figure (2) shows the sum of squared fitting errors over a prediction horizon for different values of the input signal. A quadratic approximation and several cubic polynomials are evaluated in order to see the effects of the prediction horizon length over the approximation. Notice that $T_{P}=N_{H} \times T_{s}$ represents the prediction horizon in seconds and its length is a tuning parameter for the controller having effects over the accuracy of the approximation as well. In this context, $T_{P}$ should be selected as short as possible in order to diminish the fitting error over the prediction.

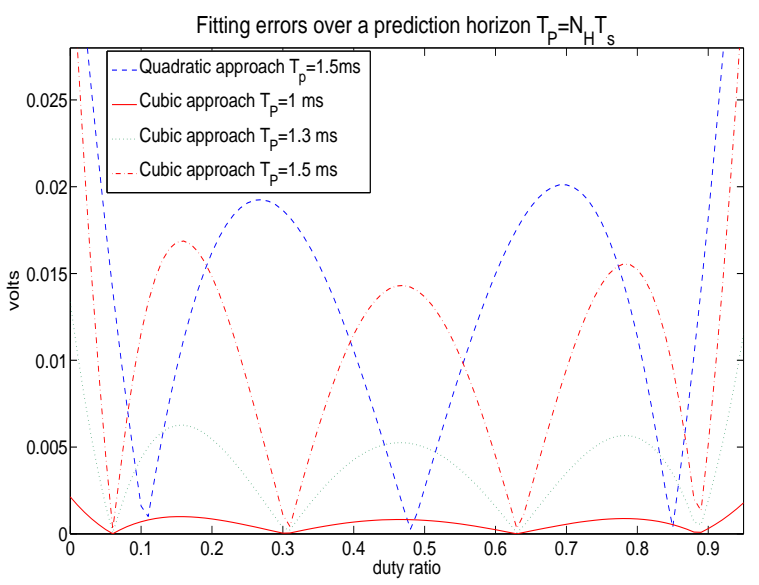

Fig. 2. Fitting errors in a prediction horizon for the input range

The cubic approximation to (4) over a prediction horizon $N_{H}=20$ is shown in (5) with $\mathbf{P}(u)$ in $\Re^{3 \times 20}$. The coefficients of the cubic polynomial are calculated using a least squares approach for the input varying from 0 to 0.95 .

$$
\begin{aligned}
\mathbf{y} & =\mathbf{P}(u) \mathbf{z} \\
\mathbf{P}(u) & =\mathbf{P}_{\mathbf{0}}+\mathbf{P}_{\mathbf{1}} u+\mathbf{P}_{\mathbf{2}} u^{2}+\mathbf{P}_{\mathbf{3}} u^{3}
\end{aligned}
$$

\subsection{The explicit control law}

The cost function for the unconstrained NMPC is formulated in terms of the prediction errors over $N_{H}$. A reference trajectory $\mathbf{r}$ can be added at this point in order to give more degrees of freedom to the design. The prediction errors $\mathbf{E}=\mathbf{r}-\mathbf{y}$ can be recast using (5) to include the dynamics of the reference filter over $N_{H}$ by defining a new set of matrices as in (6). The vectors $\mathbf{g}_{\boldsymbol{f}}$ and $\mathbf{f}_{\mathbf{f}}$ correspond respectively to the free and forced response coefficients of the first order reference filter with unit gain and time constant $\tau_{\text {ref }}$.

$$
\begin{aligned}
\mathbf{E}=\mathbf{M}(u) \mathbf{w} \\
\mathbf{w}=\left(\begin{array}{lll}
\mathbf{z}^{T} & y(t) & s p(t)
\end{array}\right)^{T} \\
\mathbf{M}_{\mathbf{0}}=\left(\begin{array}{lll}
\mathbf{P}_{\mathbf{0}} & \mathbf{g}_{\mathbf{f}} & \mathbf{f}_{\mathbf{f}}
\end{array}\right) \\
\mathbf{M}_{\mathbf{1}}=\left(\begin{array}{lll}
\mathbf{P}_{\mathbf{1}} & \mathbf{0} & \mathbf{0}
\end{array}\right) \\
\mathbf{M}_{\mathbf{2}}=\left(\begin{array}{lll}
\mathbf{P}_{\mathbf{2}} & \mathbf{0} & \mathbf{0}
\end{array}\right) \\
\mathbf{M}_{\mathbf{3}}=\left(\begin{array}{llll}
\mathbf{P}_{\mathbf{3}} & \mathbf{0} & \mathbf{0}
\end{array}\right)
\end{aligned}
$$

With the previous equations, the optimization problem is structured as in $(3)^{2}$ which is minimized using a Newton approach by solving $\mathbf{g}(u, \mathbf{w})=\mathbf{J}^{T}(u, \mathbf{w}) \mathbf{E}(u, \mathbf{w})=0$, with $\mathbf{J}=$ $\frac{\partial \mathbf{E}(u, \mathbf{w})}{\partial u}$. The calculus of the Jacobian $J$ can be executed easily due to the polynomial approximation on the prediction error.

The solution of $\mathbf{g}(u, \mathbf{w})=0$ involves a polynomial expression in $u$ of fifth order. To avoid solving it explicitly, $\mathbf{g}(u, \mathbf{w})$ is expanded in a Taylor series and solved iteratively in $u$ as shown in (7). This would correspond to one Newton iteration using the initial value embedding (Diehl et al., 2005). The terms $u_{0}$ and $\mathbf{w}_{\mathbf{0}}$ denote past values of $u$ and $\mathbf{w}$ while $u_{1}$ and $\mathbf{w}_{\mathbf{1}}$ their current values respectively.

$$
\begin{aligned}
u_{1}= & u_{0}-{\frac{\partial \mathbf{g}\left(u_{0}, \mathbf{w}_{\mathbf{0}}\right)}{\partial u}}^{-1} \mathbf{g}\left(u_{0}, \mathbf{w}_{\mathbf{0}}\right) \\
& -\frac{\partial \mathbf{g}\left(u_{0}, \mathbf{w}_{\mathbf{0}}\right)^{-1}}{\partial u} \frac{\partial \mathbf{g}\left(u_{0}, \mathbf{w}_{\mathbf{0}}\right)}{\partial \mathbf{w}}\left(\mathbf{w}_{\mathbf{1}}-\mathbf{w}_{\mathbf{0}}\right)
\end{aligned}
$$

Solving the partial derivatives in (7), it can be reformulated as in (8) where the prime represents the order of the derivative with respect to $u$.

$$
\begin{aligned}
u_{1} & =u_{0}-\left(\mathbf{w}_{\mathbf{0}}{ }^{T} \mathbf{A}_{\mathbf{g}} \mathbf{w}_{\mathbf{0}}\right)^{-1}\left(\mathbf{w}_{\mathbf{0}}^{T} \mathbf{B}_{\mathbf{g}}\left(2 \mathbf{w}_{\mathbf{1}}-\mathbf{w}_{\mathbf{0}}\right)\right) \\
\mathbf{A}_{\mathbf{g}} & =\mathbf{M}^{\prime T}\left(u_{0}\right) \mathbf{M}^{\prime}\left(u_{0}\right)+\mathbf{M}^{T}\left(u_{0}\right) \mathbf{M}^{\prime \prime}\left(u_{0}\right) \\
\mathbf{B}_{\mathbf{g}} & =\frac{\mathbf{M}^{\prime T}\left(u_{0}\right) \mathbf{M}\left(u_{0}\right)+\mathbf{M}^{T}\left(u_{0}\right) \mathbf{M}^{\prime}\left(u_{0}\right)}{2}
\end{aligned}
$$

Notice that $\mathbf{A}_{\mathbf{g}}$ and $\mathbf{B}_{\mathbf{g}}$ are polynomials like $\mathbf{M}(u)$ but with reduced dimensions on their coefficients ${ }^{3}$ due to the products of the form $\mathbf{M}^{T} \mathbf{M}$. In fact the matrices which describe $\mathbf{A}_{\mathbf{g}}$ and $\mathbf{B}_{\mathbf{g}}$ are in $\Re^{5 \times 5}$ as a consequence of the dimensions of w. Then the inversion involved in (8) corresponds to a scalar inversion avoiding computational load to the algorithm.

Equation (8) can be easily programmed by storing the matrices which correspond to coefficients of the polynomials $\mathbf{A}_{\mathbf{g}}$ and $\mathbf{B}_{\mathbf{g}}$ in memory and computing at each sampling instant the control law.

\footnotetext{
2 Using $\mathbf{w}$ instead of $\mathbf{x}$

3 However, the order of $\mathrm{Ag}$ is 4 and $\mathrm{Bg}$ is 5
} 
In order to estimate the states of the system, an Extended Kalman Filter $(E K F)^{4}$ was tuned with the model provided in (2). Table 3 summarizes the parameters used for the NMPC.

Table 3. Parameters for the NMPC

\begin{tabular}{ll}
\hline Parameter & value \\
\hline$N_{H}$ & 20 samples \\
$N_{u}$ & 1 sample \\
$\tau_{\text {ref }}$ & $1 \mathrm{~ms}$ \\
$T_{s}$ & $50 \mu \mathrm{s}$ \\
$K_{n}$ & 60 \\
$Q_{W}$ & 25 \\
$R_{W}$ & 0.5 \\
\hline
\end{tabular}

\section{EVALUATION}

The designed controllers are evaluated through disturbance and tracking tests on the hybrid model. Since the controllers where designed for the average model, a PWM unit is needed. The control algorithms deliver a value $u(t)$ in the range [0 0.95] to the PWM and it closes the switch $u \times T_{s}$ seconds during each switching period $T_{s}$.

Table 4 summarizes the changes executed to test the performance of the designed controllers. The results for the voltage, current and control efforts are illustrated in figures 3,4 and 5 respectively.

Table 4. Disturbance Test Description

\begin{tabular}{lc}
\hline Time & Change \\
\hline $10 \mathrm{~ms}$ & setpoint change \\
$30 \mathrm{~ms}$ & Input voltage decreases from $18.3 \mathrm{~V}$ to $13.3 \mathrm{~V}$ \\
$50 \mathrm{~ms}$ & Load resistor decreases $50 \%$ \\
$70 \mathrm{~ms}$ & setpoint change \\
\hline
\end{tabular}

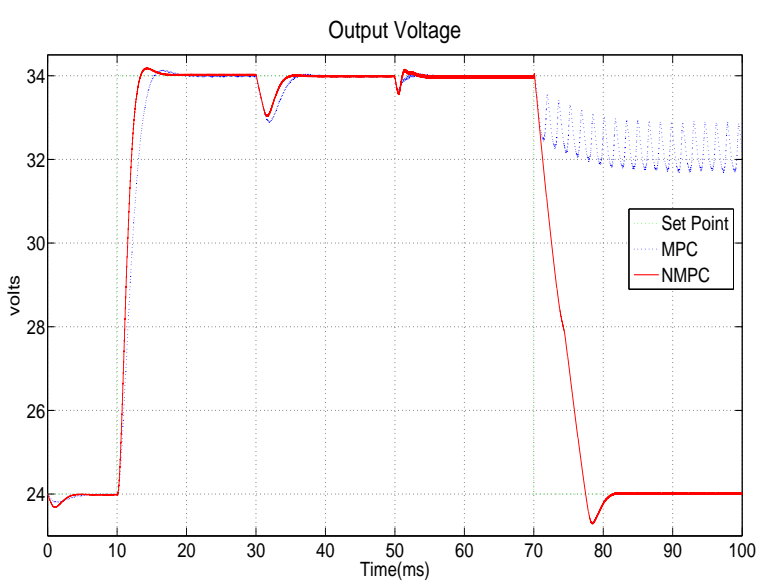

Fig. 3. Output voltage response for the disturbance test

Although the performance of both controllers is similar with respect to the disturbance rejection,

4 The reader is refereed to (Grewal and Andrews, 2001) for details on the $E K F$ implementation
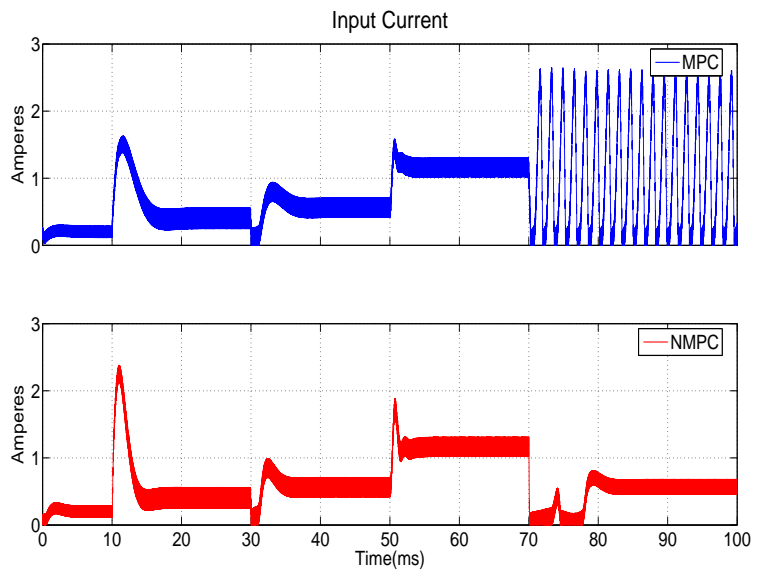

Fig. 4. Input current response for the disturbance test
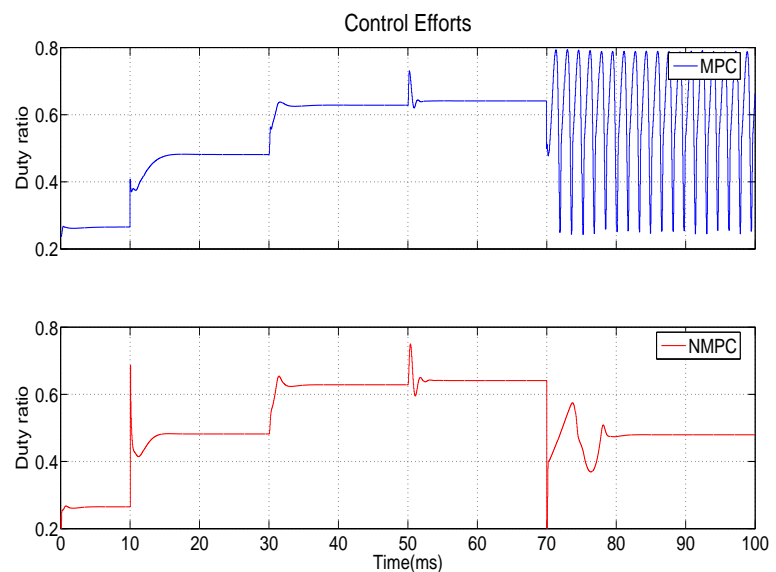

Fig. 5. Control efforts for the disturbance test

the proposed NMPC is able to deal in a better way with the DCM which brings the linear MPC to a unstable behavior.

A second test to evaluate the performance over output voltage range (18.3 to $72 \mathrm{~V}$ ) was executed. The tracking performance was tested by a series of steps in the setpoint each $10 \mathrm{~ms}$. Figures 6, 7 and 8 show the output voltage, input current and control actions respectively. The good behavior for the NMPC strategy is noticed especially for output voltages near to the maximum, where the linear technique is affected, showing some oscillations and presenting unstable behavior.

\section{CONCLUSIONS}

This work has presented a NMPC strategy for a boost DC-DC converter. The obtained performance is in general better than the one shown by linear techniques (MPC). However, the MPC tuned with a simple noise model showed an acceptable performance for the disturbance rejection test but is unable to deal with the Discontinous Conduction Mode. 


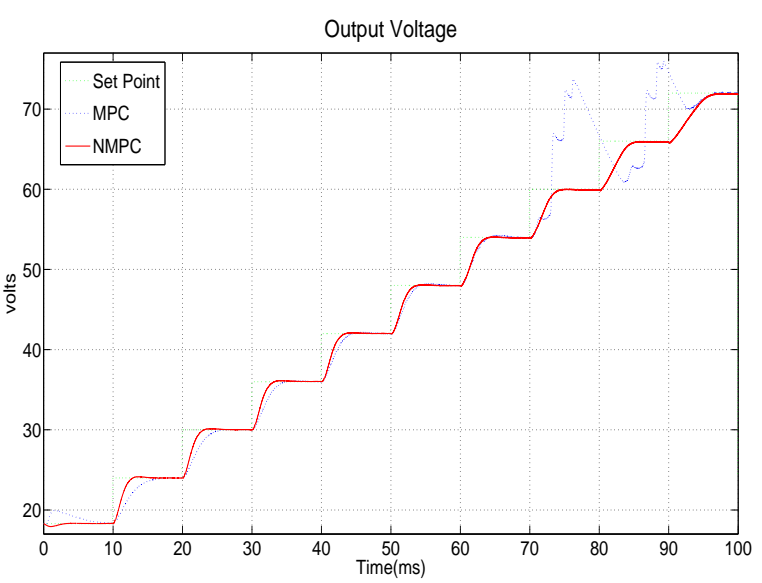

Fig. 6. Voltage response for the tracking test
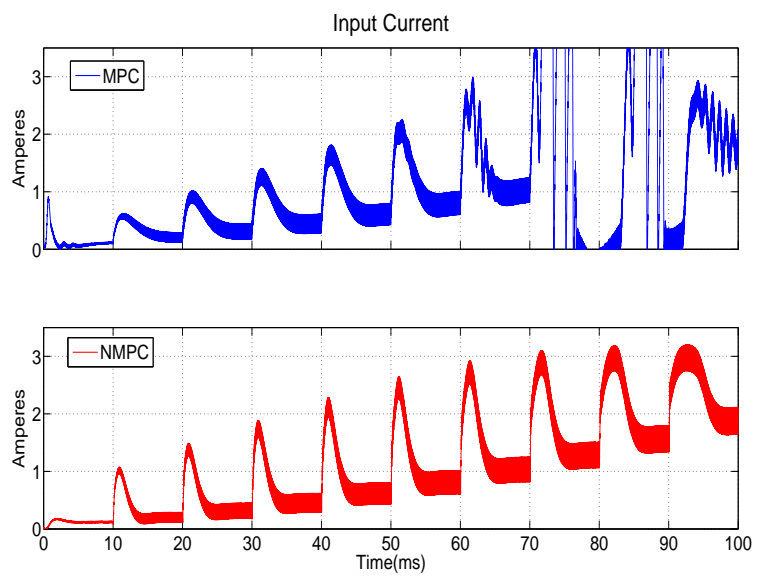

Fig. 7. Input current for the tracking test
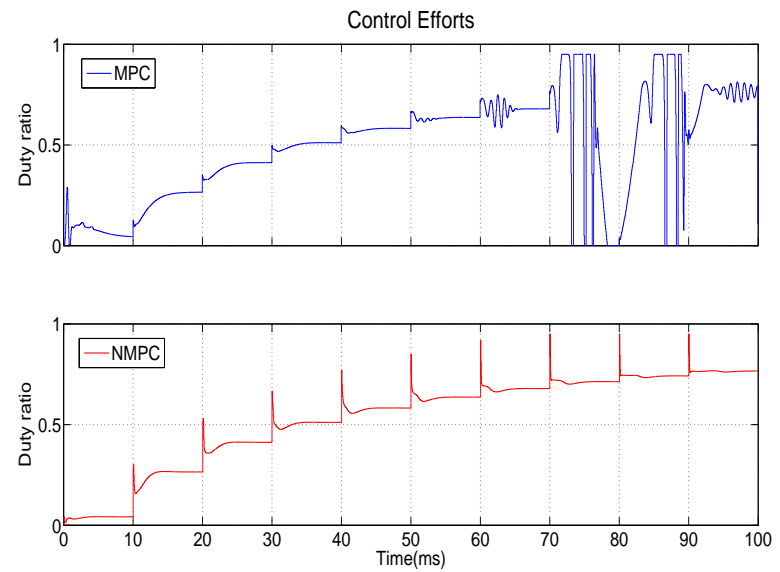

Fig. 8. Control signals for the tracking test

Although the proposed NMPC applies a suboptimal solution at each sampling instant due to the approximation of the exact solution, the simulation study showed good convergency of the algorithm to the optimal solution for the evaluated test.

Since the simulation study for the designed controllers indicates a viable solution, the next step will be to evaluate the design on a real platform.
The polynomial formulation of the solution allows an easy implementation and low computational load to the system making possible the short sampling period of $50 \mu \mathrm{s}$.

\section{ACKNOWLEDGMENT}

This work has been financially supported with: •a grant from the European Commission ALFA office, within the Project II-0407-FA, Latin American BioProcess Control, $\bullet$ Research Council KUL: GOA AMBioRICS, CoE EF/05/006 Optimization in Engineering(OPTEC), several $\mathrm{PhD} /$ postdoc \& fellow grants; - Flemish Government: FWO: $\mathrm{PhD} /$ postdoc grants, projects, G.0407.02, G.0197 .02 , G.0141.03, G.0491.03, G.0120.03, G.0452.04, G.0499.04, G.0211.05, G.0226.06, G.0321.06, G.03 02.07, research communities (ICCoS, ANMMM, MLDM); IWT: PhD Grants, McKnow-E, EurekaFlite2; • Belgian Federal Science Policy Office: IUAP P6/04 (DYSCO, Dynamical systems, control and optimization, 2007-2011); • EU: ERNSI.

\section{REFERENCES}

Diehl, M., H.G. Bock and J.P. Schlöder (2005). A real-time iteration scheme for nonlinear optimization in optimal feedback control. SIAM Journal on Control and Optimization 43(5), 1714-1736.

Escobar, G., R. Ortega, H. Sira-Ramirez, J-P. Vilain and I. Zein (1997). An experimental comparison of several nonlinear controllers for power converters.. Proceedings of the 36th IEEE Conference on Desition and Control 1, 227-228.

Geyer, T., G. Papafotiou and M. Morari (2005). Model predictive control on power electronics: A hybrid systems approach. 44th IEEE Conference on Desition and Control and the European Control Conference pp. 5606-5611.

Grewal, M. and A. Andrews (2001). Kalman Filtering, Theory and practice using Matlab. John Wiley and Sons Inc.. USA.

Kalman, R. and R. Bucy (1961). New results in linear filtering and prediction theory. Transactions of the ASME - Journal of Basic Engineering 83, 95-107.

Ramirez-Garcia, P. and R. De Keyser (2001). Model based predictive control of switched mode power supplies. Proc. of the European Control Conf. - ECC2001 pp. 3771-3776.

Silva, J. (1999). Sliding-mode control of boosttype unity powerfactor pwm rectifiers. IEEE transactions on industrial electronics 46, 594-603.

Sira-Ramirez, H. (1990). Design of pi controllers for dc-to-dc power supplies via extended linearization. Int. J. Control 51, 601-620. 